\title{
Development of a radiomic signature for predicting response to neoadjuvant chemotherapy in muscle-invasive bladder cancer
}

Ambica Parmar'; Abdul Aziz Qazi²; Audrius Stundzia ${ }^{3}$; Hao-Wen Sim ${ }^{4-7}$; Jeremy Lewin ${ }^{8}$; Ur Metser²; Martin O’Malley²; Aaron R. Hansen $^{8}$

${ }^{1}$ Division of Medical Oncology \& Hematology, Sunnybrook Health Sciences Centre, University of Toronto, Toronto, ON, Canada; ${ }^{2}$ Department of Medical Imaging, University of Toronto, Toronto, ON, Canada;

${ }^{3}$ Tomographix IP Ltd, Toronto, ON, Canada; ${ }^{4}$ NHMRC Clinical Trials Centre, University of Sydney, Sydney, Australia; ${ }^{5}$ St Vincent's Clinical School, University of New South Wales, Sydney, Australia; ${ }^{6}$ Department of Medical Oncology, The Kinghorn Cancer Centre, Sydney, Australia; ${ }^{7}$ Department of Medical Oncology, Chris O'Brien Lifehouse, Sydney, Australia; ${ }^{8}$ Division of Medical Oncology \& Hematology, Princess Margaret Cancer Centre, University of Toronto, Toronto, ON, Canada

Cite as: Parmar A, Qazi AA, Stundzia A, et al. Development of a radiomic signature for predicting response to neoadjuvant chemotherapy in muscle-invasive bladder cancer Can Urol Assoc J 2021 October 18; Epub ahead of print. http://dx.doi.org/10.5489/cuaj.7294

Published online October 18, 2021

Corresponding author: Dr. Aaron Hansen, Division of Medical Oncology \& Hematology, Princess Margaret Cancer Centre, University of Toronto, Toronto, ON, Canada; aaron.hansen@uhn.ca

$* * *$

\section{Abstract}

Introduction: Neoadjuvant chemotherapy (NAC) for muscle-invasive bladder cancer (MIBC) improves overall survival, but pathological response rates are low. Predictive biomarkers could select those patients most likely to benefit from NAC. Radiomics technology offers a novel, noninvasive method to identify predictive biomarkers. Our study aimed to develop a predictive radiomics signature for response to NAC in MIBC.

Methods: An institutional bladder cancer database was used to identify MIBC patients who were treated with NAC followed by radical cystectomy. Patients were classified into responders and non-responders based on pathological response. Bladder lesions on computed tomography images taken prior to NAC were contoured. Extracted radiomics features were used train a radial basis function support vector machine classifier to learn a prediction rule to distinguish responders from non-responders. The discriminative accuracy of the classifier was then tested using a nested 10-fold cross-validation protocol. 
Results: Nineteen patients who underwent NAC followed by radical cystectomy were found to be eligible for analysis. Of these, nine (48\%) patients were classified as responders and $10(52 \%)$ as non-responders. Nineteen bladder lesions were contoured. The sensitivity, specificity and discriminative accuracy were $52.9 \pm 9.4 \%, 69.4 \pm 8.6 \%$, and $62.1 \pm 6.1 \%$, respectively. This corresponded to an area under the curve of $0.63 \pm 0.08(\mathrm{p}=0.20)$.

Conclusions: Our developed radiomics signature demonstrated modest discriminative accuracy; however, these results may have been influenced by small sample size and heterogeneity in image acquisition. Future research using novel methods for computer-based image analysis on a larger cohort of patients is warranted.

\section{Introduction}

Neoadjuvant chemotherapy (NAC) prior to radical cystectomy (RC) in muscle-invasive bladder cancer (MIBC) has led to an improvement in 5-year overall survival (OS) rates by $5 \%$ as compared to $\mathrm{RC}$ alone, with preferential benefit for patients who achieve a pathological response (i.e. $\leq$ ypT1). ${ }^{1-4}$ However, the majority of patients fail to respond to NAC with evidence of muscle-invasive disease at the time of cystectomy. ${ }^{3}$ This low response rate, coupled with the significant risk of toxicities with recommended cisplatin-based chemotherapy regimens, raises concerns about the risk-benefit ratio of utilizing NAC in patients with MIBC particularly in patients who are older and have co-morbidities. 5,6 Accordingly, predictive biomarkers are critical to identify patients who will respond to NAC in order to limit potential toxicities from cytotoxic chemotherapy in patients who are unlikely to derive benefit.

Radiomics technology offers a promising method to identify predictive and prognostic biomarkers by extracting quantitative information from radiological imaging to describe tumor biology. ${ }^{7,8}$ As the analysis is not limited to the biopsied sample, radiomics analysis can assess for intra-tumoral heterogeneity, as well as the tumour microenvironment. ${ }^{9}$ Furthermore, as a non-invasive method it provides negligible risk to the patient and can be easily utilized to assess for tumour response to therapy over time. The potential opportunity of radiomics technology as a predictive biomarker has been demonstrated across multiple solid tumor malignancies (i.e. head and neck, lung, esophageal, rectal and testicular cancer), highlighting its potential as a predictive biomarker in MIBC. ${ }^{9-14}$

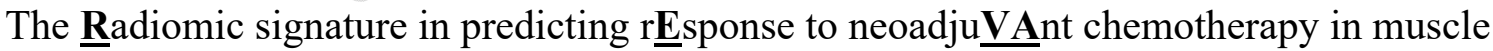
invasive bLadder cancer (REVeAL) study was thus conducted to develop and evaluate a radiomics signature that can predict the likelihood of response to NAC based on pre-treatment computed tomography (CT) for patients treated for MIBC at a single institution.

\section{Methods}




\section{Cohort creation}

All patients diagnosed with MIBC between January 1, 2001 and December 31, 2016 were identified from a retrospective review of the University Health Network Bladder Cancer database following institutional ethics approval. Eligible patients for inclusion included adult patients (age $\geq 18$ years) with biopsy proven urothelial carcinoma (squamous cell histology was excluded) who received NAC with cisplatin-based treatment. Patients were excluded if there were no computed tomography (CT) images prior to initiation of NAC and if there was no surgical pathology available from cystectomy. This cohort was divided into two sub-groups based on pathological response at the time of cystectomy: responders (i.e. pathological tumor (T) stage of $\leq \mathrm{ypT} 1$ ) and non-responders (i.e. >ypT1).

For all eligible patients, the following clinical data were extracted: a) patient demographics (i.e. age, sex), disease characteristics (i.e. TNM stage), details of neoadjuvant chemotherapy (including chemotherapy regimen, number of cycles completed), pathological response at time of cystectomy and clinical outcomes (i.e. recurrence and survival).

\section{Radiomics analysis}

Radiological contouring of the primary bladder tumour was performed manually on the pretreatment $\mathrm{CT}$ images by two reviewers in tandem (AP, AQ) using 3D Slicer software (3D Slicer version 4.10.2, available at: https://www.slicer.org/). A third investigator (AH) was available to adjudicate any disagreements. The region of interest (ROI) for image contouring was restricted to the primary bladder tumor, with exclusion of regional lymph nodes.

Quantitative radiomic features from the primary bladder lesion were extracted using computational algorithms. Each image voxel was quantized from a grey level continuum to a finite range of 32 grey levels. Each algorithm was initially described for two-dimensional (2D) pictures and then adapted for three-dimensional (3D) volumes by extrapolating from the 4 principal directions in 2D to the 13 principal directions in 3D to generate a volume of interest (VOI). ${ }^{9}$ The radiomic features were then computed in a step-wise fashion. First-order metrics specific to lesion intensity (i.e. the brightness or darkness of the imaging voxels) were computed from each VOI and classified into 11 percentiles, ranging from $0 \%$ to $100 \%$. The intensities from each VOI were then divided into 32 equal sized bins that spanned the range of image intensities between the $1^{\text {st }}$ and $99^{\text {th }}$ percentiles (percentiles chosen to minimize effect of outliers) prior to computing the 142 second-order texture metrics. The second-order texture analysis was based upon 4 classes of texture matrices that are well described in the literature: a) 13 Haralick features from the gray level co-occurrence matrix; b) 5 features from the neighborhood gray tone difference matrix; c) 10 features from the gray level run length matrix; and d) 10 features from the gray level size zone matrix. ${ }^{15-18}$ Integration of these 142 radiomics features was used to characterize the radiomics signature for responders and non-responders.

\section{Machine learning}


Each radiomics signature was defined as a responder or non-responder using the final pathological response. This information was used to train a radial basis function (RBF) support vector machine (SVM) classifier to learn a prediction rule to distinguish responders from nonresponders. ${ }^{19,20}$ In this regard, the SVM functioned as a learning model to optimally separate points within a feature space into responders versus non-responders. In the setting of ambiguity, the RBF kernel was used to map the initial feature space into a higher dimensional feature space where they ultimately become linearly separable.

In stage 1, cross validation was used to test and optimize the SVM classifier using a 10fold nested cross-validation. This approach used the same data for training and testing but in separate iterations. Nested validation allows for two loops to be performed in parallel: an inner loop (hyper-parameter tuning) which finds the optimal combination of classifier settings and the outer loop which tests the accuracy of the classifier. ${ }^{21}$ The hyper-parameters were manually set and included: 1) feature selection (fraction of the most highly associated features to retain for the prediction rule), 2) cost hyperparameter (determines the trade-off for outliers) and, 3) scale hyperparameter (determines the smoothness of the decision surface). ${ }^{22}$ For each of the 100 iterations of the outer loop, ten-fold cross-validation was used with $90 \%$ of the data used in the inner loop and $10 \%$ of the data for testing. Within the inner loop, ten-fold cross-validation protocol was employed for each point in a 3D grid covering a range of fractions of the best features to retain (feature selection), values of the cost parameter and values of the scale parameter. For each grid point searched, the inner-loop cross validation result was recorded and then the best-performing triple of hyperparameters was utilised to train a classifier using all of the inner loop data. This classifier was then applied to the $10 \%$ data held for testing, and the results recorded as the accuracy.

In stage 2, the texture features, as per the binary classification by response, were inputted into the supervised machine learning (SML) RBF SVM classifier to train for the discrimination between the responders and non-responders. The accuracy of the classifier was then tested using a nested 10-fold cross-validation protocol.

\section{Outcome measure}

Description of the accuracy of the final predictive radiomics signature from the SVM classifier was reported by its sensitivity, specificity, area under receiver operator curve (AUC) and statistical significance.

\section{Statistical analysis}

Descriptive statistics (mean, median, counts and proportions) were used to summarize the clinical characteristics of the included cohort. All data was analyzed descriptively with proportions for categorical data, or medians and interquartile ranges (IQR) for continuous data. The two patient groups were compared with the use of Pearson's Chi-squared test for all categorical data. Continuous data was compared with a one-way analysis of variance. Data for time dependent variables were reported as medians with $95 \%$ confidence intervals $(\mathrm{CI})$. The 
Kaplan-Meier method was used to estimate relapse-free survival (RFS) and OS, with corresponding $95 \%$ CI. RFS was defined as the time from pathological diagnosis to date of first local or regional recurrence, death, or censored at the date of last follow-up. OS was defined as the time from pathological diagnosis to date of death or censored at the date of last follow-up. Log-rank statistics were used to compare RFS between responders and non-responders. Statistical significance for the predictive accuracy of the radiomics signature from the SVM classifier was determined by permutation testing, at a significance level of 5 percent.

\section{Results}

\section{Study cohort}

Thirty-two patients with MIBC who underwent NAC were identified. Of these 8 patients were excluded due to lack of available pre-treatment CT images, 4 due to an inability to localize bladder tumor and 1 due to imaging artifact, leaving 19 patients eligible for further analysis. Of these, $9(48 \%)$ patients were classified as responders and $10(52 \%)$ as non-responders based on their pathology at the time of cystectomy. Table 1 summarizes the clinical characteristics and outcomes of the included cohort. The majority of patients had their pre-treatment CT imaging following pathological sampling $(n=13 ; 68 \%)$ with a median time from pathological diagnosis to CT imaging of 43 days (95\% CI: $20-106)$. Four patients (21\%) had CT imaging prior to pathological sampling and for 2 patients $(11 \%)$ the date of pathological sampling is unknown. The median time from pre-treatment CT imaging to cystectomy was 157 days (95\% CI: 128 235).

The median RFS for responders and non-responders was 51 months (95\% CI: 28 - not reached) and 24 months (95\% CI: 20 - not reached, $p=0.28$ ), respectively. (Figure 1) Median OS for the study cohort was not reached.

Among the included study cohort, a total of 19 bladder lesions, in 19 patients were analyzed. The majority of bladder tumors were visualized on contrast-enhanced CT scans ( $\mathrm{n}=18$; $95 \%)$ in the delayed $(n=13 ; 72 \%)$ or venous $(n=5 ; 28 \%)$ phase. CT images were acquired with 3 mm slice thickness for all but one patient $(n=18 ; 95 \%)$. All but one included patient had a solitary bladder lesion that was included for image analysis. One patient in the non-responding sub-group was found to have multi-focal lesions, all of which were included as one lesion in the ROI. Image analysis was restricted to the bladder lesion only. Thus, for the three patients identified to have node-positive disease, nodal disease was not included in the ROI. The anatomical location of the lesions across the cohort were found to be posterior-lateral (right: 7 (37\%), left: $3(16 \%)$ ), anterior-lateral (right: $4(21 \%)$, left: $1(5 \%)), 2$ left-lateral $(11 \%), 1$ posterior (5\%), 1 multi-focal (5\%). Median time from CT imaging to initiation of NAC for the cohort was 24 days (95\% CI: 10-43 days).

\section{Radiomics analysis}


Neuroimaging Informatics Technology Initiative (NIfTI) software libraries were used to extract lesion VOI contours. Series of lesion VOI that were specific to a subject were examined using the radiomics analysis software to confirm successful extraction of VOI contours from the NIfTI files and to confirm their positions matched those of a bladder lesion. The data set was found to be suitable for radiomics analysis. Batch processing of the total ReVEAL data generated the set of texture features that were then used by the second stage radiomics SML application.

\section{Machine learning}

The receiver-operator characteristic (ROC) curve, based upon the iterative grid over the region of highest cross-validation accuracy, is represented in Figure 2. The mean discriminative accuracy of the radiomics signature from the SVM classifier was found to be $62.1+/-6.1 \%$. The sensitivity and specificity of the radiomics signature was found to be $52.9+/-9.4 \%$ and $69.4+/-$ $8.6 \%$, respectively. This corresponded to an AUC of $0.63+/-0.08(p=0.20)$.

\section{Discussion}

The ReVEAL study aimed to develop a predictive radiomics signature to identify patients with MIBC who were likely to respond to NAC. To our knowledge, this is the first evaluation of a predictive radiomics signature in MIBC developed on pre-treatment CT images that benchmarked response as per pathological assessment. Our study demonstrated the feasibility of extracting radiomic features from pre-treatment CT imaging in $\mathrm{MIBC}$ for the development of a predictive radiomics signature. The discriminative accuracy of our predictive radiomics signature was modest at $62 \%$.

In the absence of contraindications, clinical practice guidelines recommend the use of NAC with cisplatin-based chemotherapy for patients with MIBC given data supporting a survival benefit, as compared to cystectomy alone. ${ }^{2,23}$ Despite this, utilization is low with populationlevel studies reporting less than $20 \%$ of patients with MIBC receive NAC. ${ }^{1,24}$ Due to the advanced age, and high rate of co-morbid conditions among patients with $\mathrm{MIBC}$, it is postulated the low use of the NAC may be due to concerns for the risk of toxicities, coupled with an expected low benefit. ${ }^{5,6}$ As there is a preferential survival benefit from NAC seen among patients who achieve a pathological complete response, foregoing NAC may be appropriate in the $40-50 \%$ of patients who ultimately fail to receive benefit. ${ }^{3,25}$ However, with current evidence demonstrating the disproportionately low rates of NAC use, it is evident that there is likely a substantial proportion of patients in which curative treatment is underutilized. As such, there is a critical need for predictive biomarkers to allow for better precision in treatment decision-making.

Molecular profiling has been investigated for its use as a predictive biomarker for response to NAC. In particular, alterations in the DNA repair pathways have demonstrated potential in this regard, with loss of function alterations in ATM, RB1, FANCC, ERCC2 and $B R C A 1$ being shown to predict pathological response and improvements in survival. ${ }^{26-29}$ Indeed, these genomic observations are consistent with the genotoxic mechanism of action of cisplatin, which is the backbone of NAC regimens. Molecular sub-typing based upon gene expression 
profiling has also generated enthusiasm for predicting response to NAC. ${ }^{30-33}$ Although early reports have demonstrated preferential response to NAC across the different molecular subtypes, they have not yet been validated as a predictive biomarker and would be challenging to integrate in routine clinical care for multiple reasons. Molecular subtyping may require additional tissue acquisition by cystoscopy with risk-associated biopsies, which are subject to sampling errors; and the technology and expertise required to perform such analyses are usually restricted to research focused academic laboratories.

There has been a growing interest in the application of radiomics technology in oncology over the past decade. The early success of computer-based image analysis for clinical prediction has been established across a range of solid tumor malignancies. For instance, image analysis to predict histology and to identify occult metastases, such as in regional lymph nodes, has shown early signs of promise. ${ }^{34,35}$ In addition, there is data supporting the use of this technology as a predictive biomarker in breast cancer, esophageal carcinoma, germ-cell tumors, nasopharyngeal carcinoma and rectal cancer, with discriminative accuracies greater than 70\%. ${ }^{9,10,14,36-38}$ To date, computer-based image analysis has not been shown to out-perform validated clinical prediction tools, in settings whereby such tools exist; however, their combination with clinical predictors have been shown to improve discriminative accuracy. ${ }^{36}$ The utility of this technology in these early applications underscore the need for further study, particularly in settings where clinical predictors alone are ineffective.

Radiomics technology has the potential to serve as a much needed predictive biomarker for NAC in MIBC. As there is no pathological assessment required, the need for additional invasive procedures is avoided thereby minimizing risks to patients and also reducing healthcare resource utilization. Similarly, as patients routinely undergo radiological imaging prior to their treatment, no additional diagnostic imaging is required. As conducted through computer-based image analysis, the need for additional healthcare personnel to facilitate this predictive biomarker is greatly reduced. Consequently, the use of radiomics technology in this context promotes the most efficient use of our healthcare resources.

The use of radiomics technology as a predictive biomarker has been previously evaluated in MIBC. ${ }^{39,40}$ Cha et al evaluated the prediction accuracy of a radiomics signature on NAC treatment response as assessed by pre- and post-treatment $\mathrm{CT}$ images, with response classified as T0 tumor on post-treatment imaging. With over 80 patients in each of their training and validation cohorts, they demonstrated their radiomic signature to have an AUC of $0.69 .{ }^{40}$ In a later study, Cha et al evaluated a computerized-based decision-support system for treatment response that used a combination of both deep-learning neural networks and radiomic technology among 123 patients with response to NAC assessed by post-treatment CT imaging. In this analysis, the authors reported an AUC of 0.80, which outperformed clinician visual assessment alone (AUC 0.74). ${ }^{39}$ Although these two studies demonstrate the promise of radiomics to predict response to NAC, the use of post-treatment CT images as a surrogate for pathological response was an important limitation, as radiological response rate does not always correlate with 
pathological outcomes. ${ }^{41}$ Unlike these prior studies in MIBC, the REVeAL study benchmarked response to treatment as per pathological assessment.

Our study is not without its limitations. Our radiomics signature was developed on a small pilot cohort of patients from a single institution, which is among the smaller sample sizes of radiomics analysis in the published literature. ${ }^{39-41}$ This small sample size limits the ability to make any conclusions on the utility of radiomics analysis as predictive biomarker for response to NAC in MIBC. The retrospective design of this study led to variations in the images used in the analysis. For instance, not all images were obtained with intravenous contrast with additional variations in the $\mathrm{CT}$ scanners and imaging protocols that introduced differences with respect to image resolution. Further, heterogeneity in the distensions of the bladder lesions could have introduced uncontrollable variation in the image analysis. In addition, the retrospective nature of this study also introduced the potential for time variation as time of imaging prior to NAC was variable. Furthermore, the small sample size necessitated the use of a cross-validation approach, as opposed to independent validation on a separate cohort of patients. However, this approach is established in the machine learning literature. ${ }^{42}$ Finally, the current study lacks a comparative analysis to address how the radiomics signature performs as compared with, or to complement, clinician prediction and/or molecular profiles.

Although the utility of our radiomics signature was limited, this represents a starting point for further evaluation of radiomics in MIBC. As the field of radiomics rapidly evolves, there is opportunity to introduce novel methods to improve the performance of the radiomics signature. This may include the use of more advanced supervised machine learning such as with convolutional neural networks that learn predictive features in a more automated fashion and have been shown to improve diagnostic accuracy. ${ }^{43}$ In addition, methods such as unsupervised deep learning, automated volume delineation or variational auto-encoders that allow for automated image analysis will allow for image analysis across a larger cohort of patients leading to data that more confidently could inform treatment decision-making in MIBC.

\section{Conclusions}

Through the ReVEAL study, we demonstrated the feasibility of developing a predictive radiomics signature to discriminate response to NAC for patients with MIBC that demonstrated modest accuracy. Future research using novel methods for computer-based image analysis on a larger cohort of patients is planned to improve the utility of radiomics as a predictive biomarker in MIBC.

Conflicts of interest: Dr. Sim has received research funding from AbbVie and Bristol-Myers Squibb. Dr. Metser has served in a consulting or advisory role or Point Biopharm Inc. Dr. Hansen has received honoraria from AstraZeneca/MedImmune, Bristol-Myers Squibb, GlaxoSmithKline, Merck, Novartis, and Pfizer; has served in a consulting or advisory role for Boehringer Ingelheim, Boston Biomedical, Bristol-Myers Squibb, GlaxoSmithKline, Merck, Novartis, and Roche/Genetech; and has received research funding from Bristol-Myers Squibb, Boehringer 
Ingelheim, GlaxoSmithKline, Janssen, Karyopharm Therapeutics, Merck, Novartis, and Roche/Genetech. The remaining authors report no personal or financial conflicts related to this work.

\section{References}

1. Booth CM, Siemens DR, Li G, et al: Perioperative chemotherapy for muscle-invasive bladder cancer: A population-based outcomes study. Cancer 120:1630-8, 2014

2. Grossman HB, Natale RB, Tangen CM, et al: Neoadjuvant chemotherapy plus cystectomy compared with cystectomy alone for locally advanced bladder cancer. N Engl J Med 349:859-66, 2003

3. Lee FC, Harris W, Cheng HH, et al: Pathologic Response Rates of Gemcitabine/Cisplatin versus Methotrexate/Vinblastine/Adriamycin/Cisplatin Neoadjuvant Chemotherapy for Muscle Invasive Urothelial Bladder Cancer. Adv Urol 2013:317190, 2013

4. Winquist E, Kirchner TS, Segal R, et al: Neoadjuvant chemotherapy for transitional cell carcinoma of the bladder: a systematic review and meta-analysis. J Urol 171:561-9, 2004

5. Bellmunt J, Mottet N, De Santis M: Urothelial carcinoma management in elderly or unfit patients. EJC Suppl 14:1-20, 2016

6. Galsky MD, Hahn NM, Rosenberg J, et al: Treatment of patients with metastatic urothelial cancer "unfit" for Cisplatin-based chemotherapy. J Clin Oncol 29:2432-8, 2011

7. Lambin P, Rios-Velazquez E, Leijenaar R, et al: Radiomics: extracting more information from medical images using advanced feature analysis. Eur J Cancer 48:441-6, 2012

8. Kumar V, Gu Y, Basu S, et al: Radiomics: the process and the challenges. Magn Reson Imaging 30:1234-48, 2012

9. Tixier F, Le Rest CC, Hatt M, et al: Intratumor heterogeneity characterized by textural features on baseline 18F-FDG PET images predicts response to concomitant radiochemotherapy in esophageal cancer. J Nucl Med 52:369-78, 2011

10. Zhang H, Tan S, Chen W, et al: Modeling pathologic response of esophageal cancer to chemoradiation therapy using spatial-temporal $18 \mathrm{~F}-\mathrm{FDG}$ PET features, clinical parameters, and demographics. Int J Radiat Oncol Biol Phys 88:195-203, 2014

11. Yip SS, Coroller TP, Sanford NN, et al: Relationship between the Temporal Changes in Positron-Emission-Tomography-Imaging-Based Textural Features and Pathologic Response and Survival in Esophageal Cancer Patients. Front Oncol 6:72, 2016

12. van Rossum PS, Fried DV, Zhang L, et al: The Incremental Value of Subjective and Quantitative Assessment of 18F-FDG PET for the Prediction of Pathologic Complete Response to Preoperative Chemoradiotherapy in Esophageal Cancer. J Nucl Med 57:691700, 2016

13. Cook GJ, Yip C, Siddique M, et al: Are pretreatment 18F-FDG PET tumor textural features in non-small cell lung cancer associated with response and survival after chemoradiotherapy? J Nucl Med 54:19-26, 2013

14. Metser U: Multiparameteric PET-MR assessment of response to neoadjuvant chemoradiotherapy in locally advanced rectal cancer: PET, MR, PET-MR and tumor texture analysis: a pilot study. Adv Mol Imag 5:49-60, 2015

15. Haralick R: Textural features for image classification. IEEE Transactions on Systems, Man and Cybernetics 3 610-621, 1973 
16. Lou HH: The analysis of natural textures using run length features. IEEE Transactions on Industrial Electronics 35:323-328, 1988

17. Thibault G: Texture indexes and gray level size zone matrix: application to cell nuclei classification. Pattern Recognition and Information Processing:140-145., 2009

18. M. A: Textural features corresponding to textural properties. . IEEE Transactions on Systems, Man and Cybernetics 19:1264-1274, 1989

19. Chang CC: A library for support vector machines. ACM Trans Intell Syst Technol 2:127, 2011

20. Boser BE: A training algorithm for optimal margin classifiers. . Proceedings of the fifth annual workshop on computational learning theory Pittsburgh, Pennsylvania, USA, 1992

21. Cawley GC: On over-fitting in model selection and subsequent selection bias in performance evaluation. J Mach Learn Res 11:2079-2107, 2010

22. Parmar C, Grossmann P, Bussink J, et al: Machine Learning methods for Quantitative Radiomic Biomarkers. Sci Rep 5:13087, 2015

23. Kulkarni GS, Black PC, Sridhar SS, et al: Canadian Urological Association guideline: Muscle-invasive bladder cancer. Can Urol Assoc J:230-238, 2019

24. Zaid HB, Patel SG, Stimson CJ, et al: Trends in the utilization of neoadjuvant chemotherapy in muscle-invasive bladder cancer: results from the National Cancer Database. Urology 83:75-80, 2014

25. Lane G, Risk M, Fan Y, et al: Persistent muscle-invasive bladder cancer after neoadjuvant chemotherapy: an analysis of Surveillance, Epidemiology and End ResultsMedicare data. BJU Int 123:818-825, 2019

26. Plimack ER, Dunbrack RL, Brennan TA, et al: Defects in DNA Repair Genes Predict Response to Neoadjuvant Cisplatin-based Chemotherapy in Muscle-invasive Bladder Cancer. Eur Urol 68:959-67, 2015

27. Van Allen EM, Mouw KW, Kim P, et al: Somatic ERCC2 mutations correlate with cisplatin sensitivity in muscle-invasive urothelial carcinoma. Cancer Discov 4:1140-53, 2014

28. Liu D, Plimack ER, Hoffman-Censits J, et al: Clinical Validation of Chemotherapy Response Biomarker ERCC2 in Muscle-Invasive Urothelial Bladder Carcinoma. JAMA Oncol 2:1094-6, 2016

29. Font A, Taron M, Gago JL, et al: BRCA1 mRNA expression and outcome to neoadjuvant cisplatin-based chemotherapy in bladder cancer. Ann Oncol 22:139-44, 2011

30. Seiler R, Ashab HA, Erho N, et al: Impact of Molecular Subtypes in Muscle-invasive Bladder Cancer on Predicting Response and Survival after Neoadjuvant Chemotherapy. Eur Urol, 2017

31. Kardos J, Chai S, Mose LE, et al: Claudin-low bladder tumors are immune infiltrated and actively immune suppressed. JCI Insight 1:e85902, 2016

32. Choi W, Porten S, Kim S, et al: Identification of distinct basal and luminal subtypes of muscle-invasive bladder cancer with different sensitivities to frontline chemotherapy. Cancer Cell 25:152-65, 2014

33. Cancer Genome Atlas Research N: Comprehensive molecular characterization of urothelial bladder carcinoma. Nature 507:315-22, 2014 
34. Coroller TP, Agrawal V, Huynh E, et al: Radiomic-Based Pathological Response Prediction from Primary Tumors and Lymph Nodes in NSCLC. J Thorac Oncol 12:467476, 2017

35. Wu W, Parmar C, Grossmann P, et al: Exploratory Study to Identify Radiomics Classifiers for Lung Cancer Histology. Front Oncol 6:71, 2016

36. Lewin J, Dufort P, Halankar J, et al: Applying Radiomics to Predict Pathology of Postchemotherapy Retroperitoneal Nodal Masses in Germ Cell Tumors. JCO Clin Cancer Inform 2:1-12, 2018

37. Liu J, Mao Y, Li Z, et al: Use of texture analysis based on contrast-enhanced MRI to predict treatment response to chemoradiotherapy in nasopharyngeal carcinoma. J Magn Reson Imaging 44:445-55, 2016

38. Ahmed A, Gibbs P, Pickles M, et al: Texture analysis in assessment and prediction of chemotherapy response in breast cancer. J Magn Reson Imaging 38:89-101, 2013

39. Cha KH, Hadjiiski LM, Cohan RH, et al: Diagnostic Accuracy of CT for Prediction of Bladder Cancer Treatment Response with and without Computerized Decision Support. Acad Radiol 26:1137-1145, 2019

40. Cha KH, Hadjiiski L, Chan HP, et al: Bladder Cancer Treatment Response Assessment in CT using Radiomics with Deep-Learning. Sci Rep 7:8738, 2017

41. Fukui T, Matsui Y, Umeoka S, et al: Predictive value of radiological response rate for pathological response to neoadjuvant chemotherapy and post-cystectomy survival of bladder urothelial cancer. Jpn J Clin Oncol 46:560-567, 2016

42. Petrick N, Sahiner B, Armato SG, 3rd, et al: Evaluation of computer-aided detection and diagnosis systems. Med Phys 40:087001, 2013

43. Truhn D, Schrading S, Haarburger C, et al: Radiomic versus Convolutional Neural Networks Analysis for Classification of Contrast-enhancing Lesions at Multiparametric Breast MRI. Radiology 290:290-297, 2019

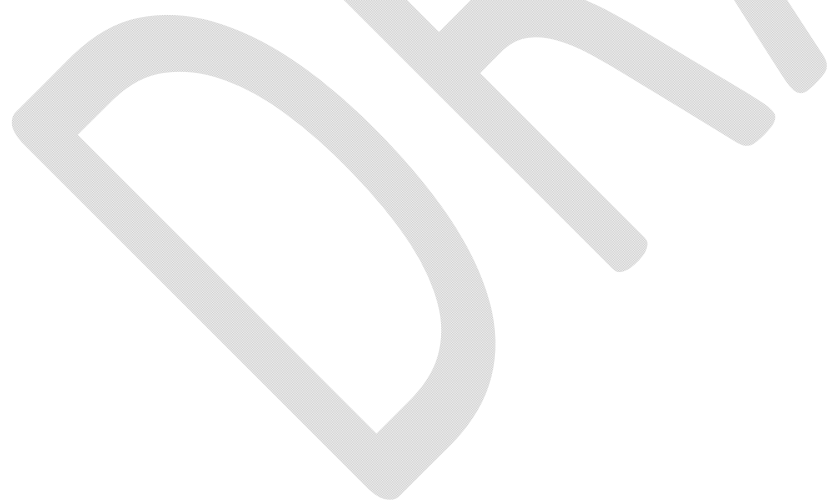




\section{Figures and Tables}

Fig. 1. Relapse-free survival for included cohort by subgroup.
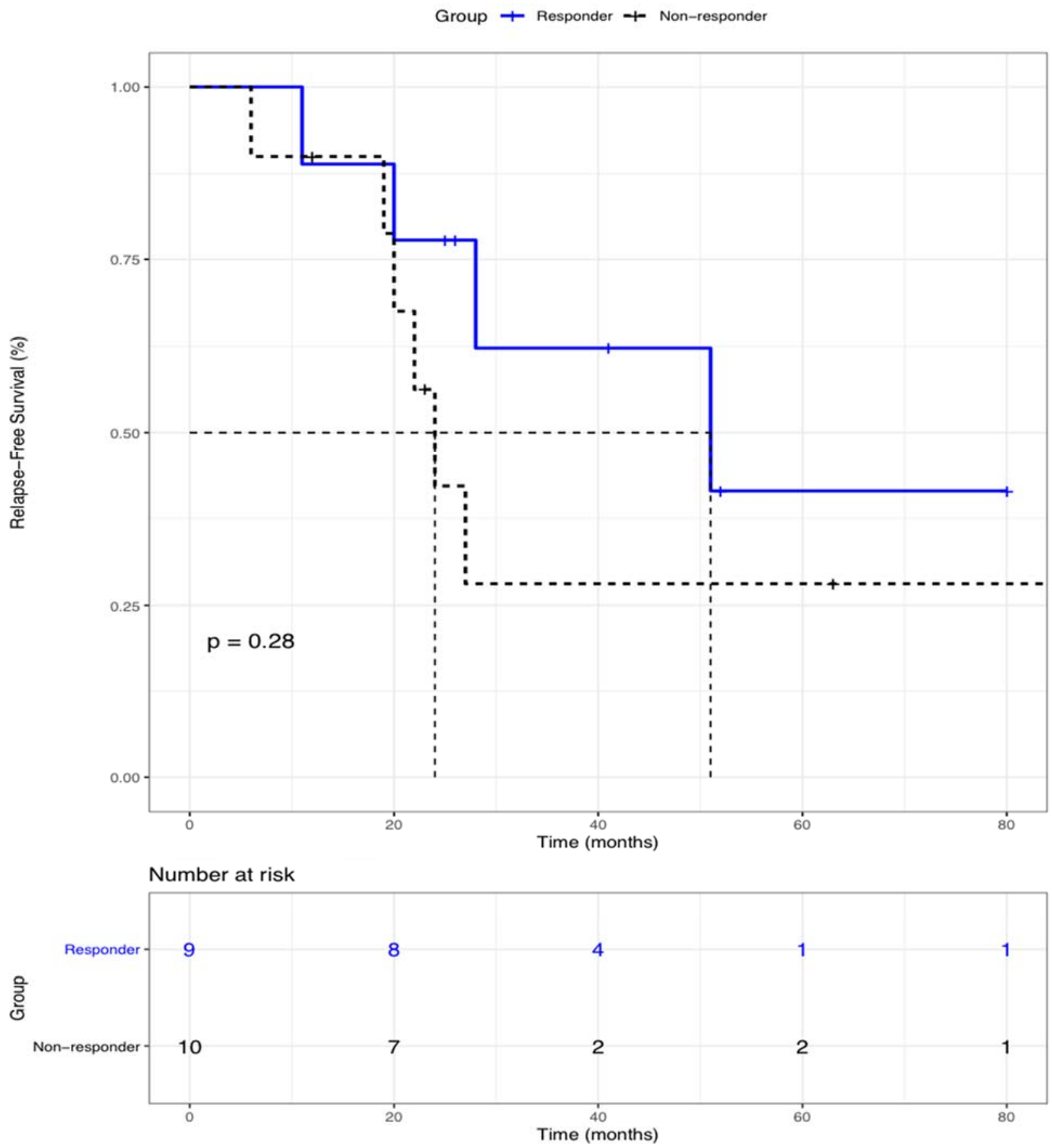
Fig. 2. Receiver operating characteristic curve for radiomic classifier for discriminating responders from non-responders on pre-treatment computed tomography images.

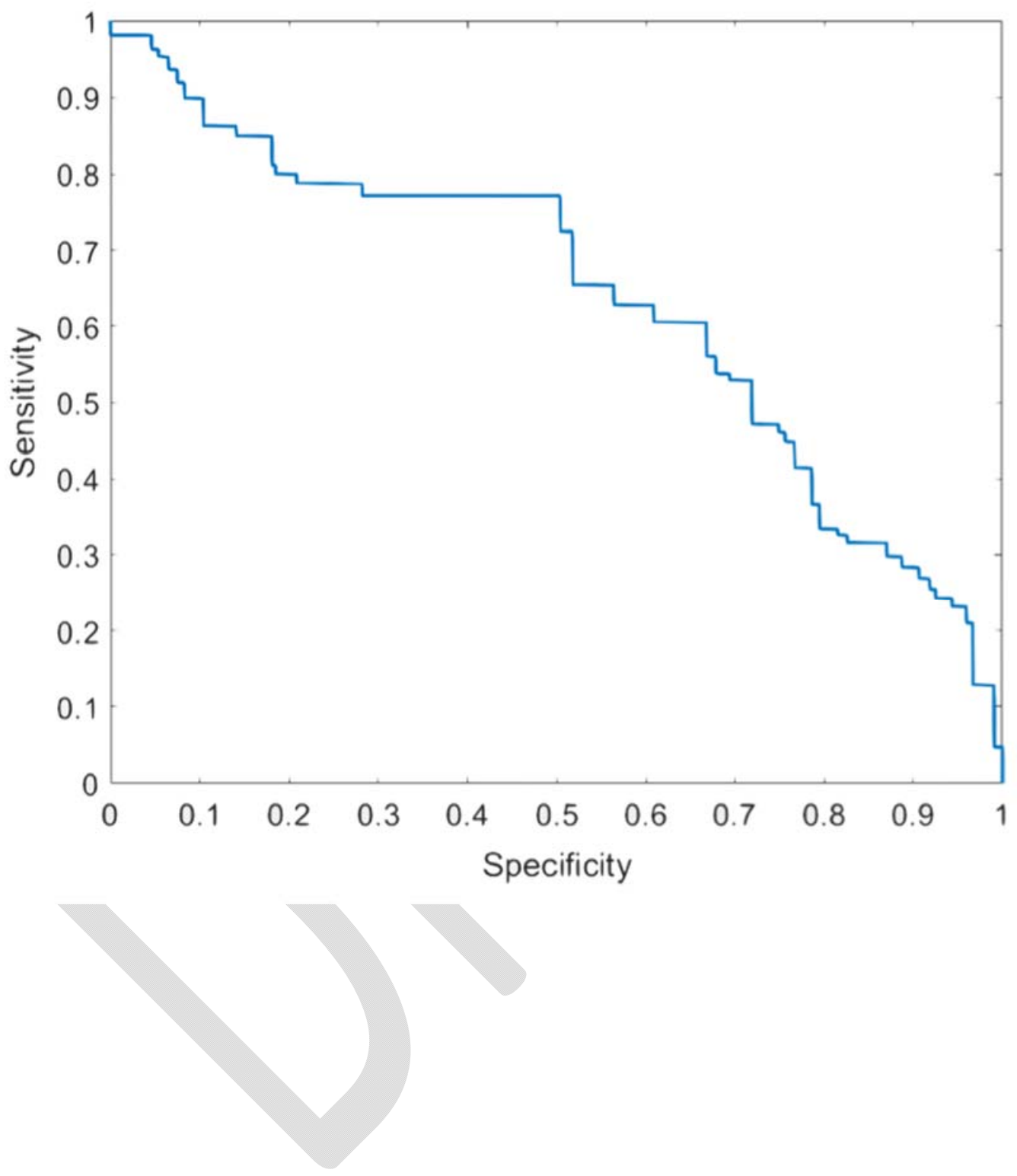




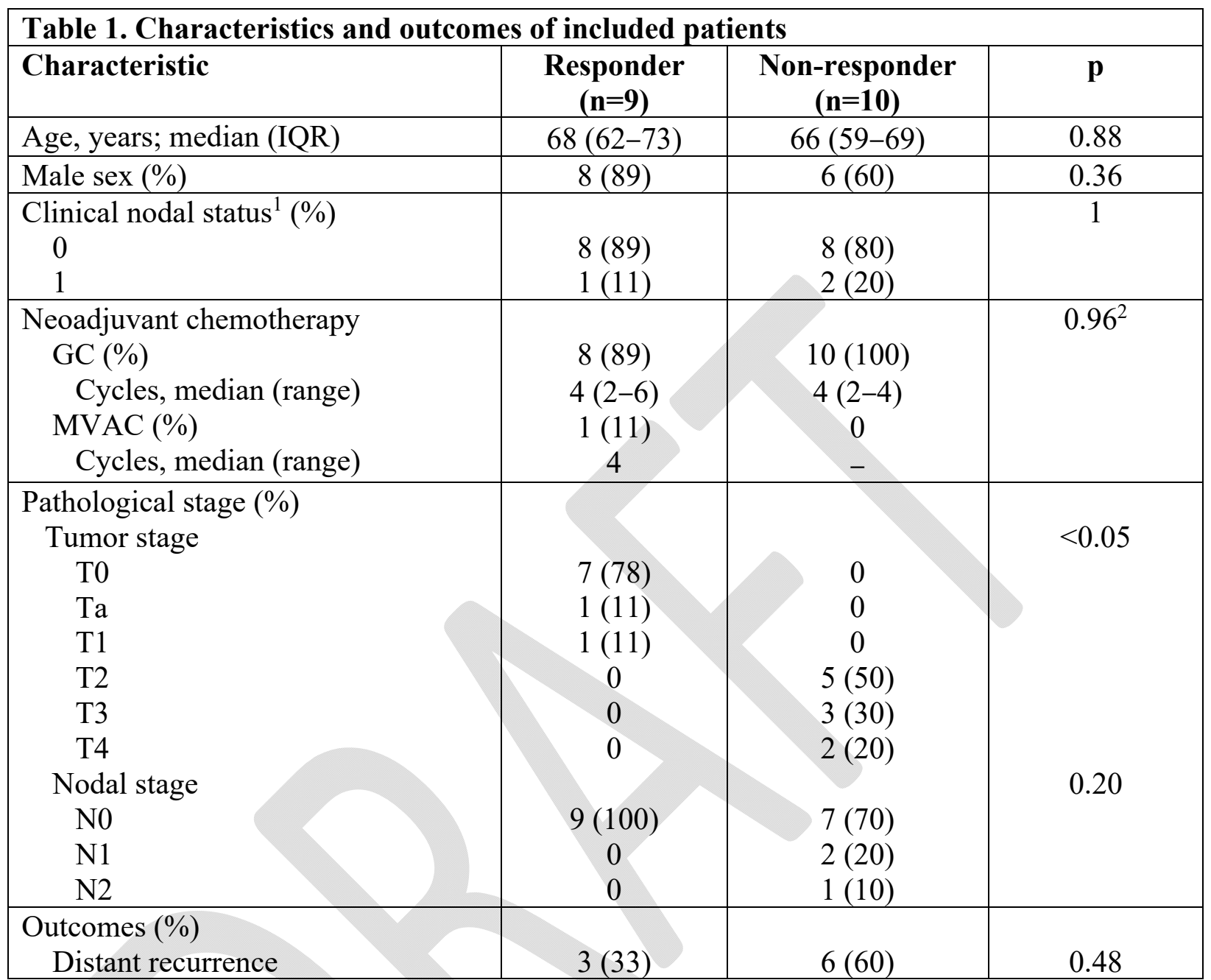

Aside from the expected difference in pathological stage, no statistically significant differences were noted between the two groups. ${ }^{1}$ Nodal status was determined on pre-treatment computed tomography imaging completed prior to neoadjuvant chemotherapy. ${ }^{2}$ Statistical testing completed on proportion of patients who received each chemotherapy regimen.

GC: gemcitabine-cisplatin; IQR: interquartile range; MVAC: methotrexate-vinblastinedoxorubicin-cisplatin. 